\title{
Prévention de la dépendance : Une nécessité de santé publique, un devoir pour la médecine gériatrique
}

\author{
B.Vellas \\ (C) Springer-Verlag France 2011
}

Trois données essentielles justifient pleinement le concept de prévention de la dépendance:

- La dépendance du sujet âgé n'est pas un état de fait, mais souvent le résultat d'un processus physiopathologique, les anglosaxons parlent de « disability process » qui peu à peu accompagne le processus de vieillissement et le transforme en un processus d'incapacités qui mène à la dépendance. Il est essentiel d'en comprendre les mécanismes physiopathologiques, cellulaires, moléculaires mais aussi sociétaux pour mieux le combattre. Avant le stade de dépendance, ce disabilty process, va entrainer un état de fragilité, qui a été négligé par notre pratique médicale. Ce sont pourtant les personnes âgées fragiles et préfragiles, de nos jours environ $40 \%$ des plus de 65 ans qui sont le plus à risque de dépendance alors qu'à ce stade de fragilité, la prévention y est encore possible. Ces personnes âgées préfragiles ou fragiles ne sont, de nos jours, ni identifiées, ni réellement prises en compte de façon spécifique par notre système de santé.

- L'essentiel de la prise en charge des personnes âgées dépendantes est réalisée de nos jours, de façon empirique et non pas, selon la médecine basée sur les preuves (Evidence Based Medicine). Avant de prendre un médicament, il nous est important d'en connaitre de façon précise quelles en sont les indications, la population cible, le réel impact, les risques d'effets secondaires. La prise en charge des personnes âgées dépendantes repose le plus souvent sur des habitudes, des dogmes, des expériences certes, mais sans véritable évaluation de leur impact réel.

- C'est aujourd'hui le moment de mettre en place de grandes études interventionnelles sur la prévention de la dépendance et sur le ralentissement de sa progression. Nous avons conduit l'étude GuidAge (premier essai de prévention de la maladie d'Alzheimer en Europe) sur 2800 patients de plus de 70 ans suivis pendant 5 ans. Nous réalisons actuellement l'étude MAPT sur 1680 patients pré-fragiles afin d'étudier l'impact d'une prise en charge multidomaine associant exercices cognitifs, exercices

B. Vellas $(\bowtie)$

Président de l'IAGG

Inserm U 1027, Gerontopole, CHU Toulouse.

vellas.b@chu-toulouse.fr physiques, nutrition dans la prévention du déclin cognitif, des incapacités physiques et de la dépendance. Cing cents de ces sujets bénéficient d'une IRM cérébrale et de l'étude des plaques amyloïdes, ce qu'on appelait auparavant « plaques séniles » à l'aide du TEP F 18 et nous pourrons bientôt mieux connaitre leurs rôles et la façon de les faire disparaître ou d'en limiter les conséquences. De nouveaux traitements, notamment des anticorps monoclonaux sont en train d'être évalués par des protocoles.

Des programmes d'envergure doivent être mis en place à titre d'exemple voici ci dessous quelques programmes qui font parti du projet d'IHU sur les sciences du vieillissement et de la prévention de la dépendance que nous comptons mettre en place non seulement à Toulouse mais avec le concours de différent centre de gériatrie en France.

- Le premier va se faire en population générale, dans un canton rural d'environ 3000 habitants, à Labastide Murat, dans le Lot. Nous allons évaluer de façon systématique tous les sujets de plus de 65 ans avec des mesures très simples tels les critères de Fried dont la vitesse de marche (Un sujet qui met plus de 4 secondes pour marcher $4 \mathrm{~m}$, est à risque de dépendance. (un $\mathrm{m} / \mathrm{s}$ est en général la vitesse nécessaire pour traverser une rue pendant la durée d'un feu rouge). Nous allons ainsi définir au sein de ce canton rural les PA en bon état de santé, les pré-fragiles ou fragiles puis celles qui sont dépendantes. Nous allons pour chacune de ces catégories, à l'échelle de la population, implémenter des programmes de prévention, leur acceptabilité et dans un deuxièmes temps l'impact en terme de prévention de la dépendance, de qualité de vie, de coût pour la santé.

- Le deuxième programme va porter sur les personnes âgées qui vivent à domicile et qui commencent à recevoir l'APA car elles ont un degré de dépendance correspondant au GIR 4 qui nécessite la mise en place d'aides le plus souvent assistance à domicile. Nous avons pu observer que $50 \%$ de ces sujets vont, deux ans après, présenter une dépendance sévère, GIR 1 et 2 . Il est indispensable de concevoir, mettre en place et valider l'impact d'une prise en charge de ces patients en rupture avec les pratiques habituelles, qui ne sont d'ailleurs pas réellement définies mais approximatives. Nous avons ainsi élaboré et validé un programme d'intervention :

- Avec le médecin traitant, en développant des RCP afin de mieux identifier, comprendre et traiter les pathologies 
responsables de cette dépendance débutante,

- Travailler avec les aides à domicile et les soignants afin de repérer tôt les signes de fragilité, les suivre de façon régulière, en faisant évoluer les métiers des aides à domicile, qui pourraient très bien assurer la mesure du poids de ces sujets fragiles, de la vitesse de marche, et pourquoi pas, débuter des interventions associant exercices physiques, cognitifs, par des programmes à la portée de tous, mais validés de façon scientifique

- Les nouvelles technologies, la e-santé sont une partie intégrante de ce programme afin de mettre en lien les aides à domiciles, les familles avec les médecins, les professionnels de santé.

- Le troisième porte sur la prévention de la dépendance iatrogène (voir article C Lafont dans ce numero) :

- L'hospitalisation représente la première cause de déclin fonctionnel chez les personnes âgées : 30 à $60 \%$ des patients âgés perdent de l'autonomie sur les activités de base de la vie quotidienne (ADL) au décours d'une hospitalisation. Cette perte d'indépendance résulte de l'affection aigue qui a motivé l'hospitalisation mais est aussi liée aux modalités de prise en charge. Nous connaissons tous les infections nosocomiales, que chacun d'entre nous peut contracter au décours d'une hospitalisation. Il a fallu plusieurs décennies pour l'admettre, en mesurer la fréquence et mettre en place les actions de prévention et qualité de soins nécessaires. Nous "fabriquons" aussi de la dépendance non seulement à l'hôpital mais au domicile ou en maison de retraite. C'est une évidence pour chacun d'entre nous. Qui ne connaît pas une personne âgée qui a été hospitalisée pour une pathologie banale et qui est sortie moins bien qu'elle était entrée. Il n'est pas question ici d'accuser qui que ce soit, mais il faut que nos hôpitaux s'adaptent au vieillissement de la population des sujets qui y sont soignés. Nous avons décidé lors d'un groupe de travail international réunissant une trentaine d'experts européens et américains, sous l'égide de la Société Mondiale de Gérontologie et Gériatrie d'admettre, cette évidence, de lui donner un nom « la dépendance iatrogène » c'est à dire la dépendance évitable qui peut être induite par les soins. La Conférence Nationale de Santé a défini la iatrogénie comme « toute pathogénie d'origine médicale au sens large, compte tenu de l'état de l'art à un moment donné, qui ne préjuge en rien d'une erreur, d'une faute ou d'une négligence ». En prenant en compte l'ensemble des actes de soins, cette définition présente l'intérêt de ne pas limiter le champ de la iatrogénie aux seuls médicaments. Elle a également l'avantage de dissocier la iatrogénie de la notion de faute ou de responsabilité.

- La dépendance iatrogène fait intervenir trois composantes intriquées et cumulatives : 1) la fragilité préexistante du patient, 2) la sévérité de la pathologie qui a conduit le patient à l'hôpital 3) enfin la structure hospitalière et les soins qu'elle dispense.

- La prévention de la « iatrogenic disability » implique des étapes successives. La première, nous l'avons vue, consiste à prendre conscience que l'hospitalisation peut créer de la dépendance. La deuxième étape consiste à considérer que le déclin fonctionnel n'est pas une fatalité.

- Les interventions devront non seulement repérer les patients à risque pour qu'ils bénéficient d'une prise en charge spécialisée mais aussi avoir pour cible la structure hospitalière et les soins. Ceci implique de repenser l'organisation de l'hôpital autour de la personne âgée.

La notion de dépendance iatrogène maintenant identifiée, nous allons en mesurer sa fréquence, quelle est-elle ? $5,10,15$, $30 \%$ ? Nous allons ainsi évaluer tous les sujet de +75 ans hospitalisés dans le CHU de Toulouse, 4 CHU de France par son activité, pendant une période donnée, évaluer le niveau d'autonomie du patient à l'arrivée dans les services, avec ses proches ce qu'était son niveau 15 jours environ avant l'hospitalisation, et enfin de façon prospective lors de la sortie. Nous connaitrons alors le pourcentage de sujets qui présentent une perte d'autonomie pendant l'hospitalisation. Il nous faudra ensuite reprendre chaque dossier pour voir ce qui est responsable de ce déclin fonctionnel, les pathologies en cours ou notre prise en charge insuffisamment adaptée aux personnes âgées fragiles, de plus en plus nombreuses.

\section{Prévenir la progression de la dépendance en EHPAD}

Malgré l'existence de plus de 10000 EHPAD en France, il n'y avait pratiquement pas de recherche clinique, alors que c'est là que sont les patients les plus sévères. Or sans recherche clinique, il ne peut y avoir de véritable amélioration de la pratique clinique. C'est ainsi que nous avons créé le réseau RHEPA qui regroupe environ 400 établissements dont les équipes se sont engagées pour participer à des programmes de recherche clinque . Plus de 200 de ces établissements sont dans la région Midi Pyrénées. Nous allons mettre en place, avec ce réseau, un vaste programme d'implémentation des critères de qualité de la prise en charge en EHPAD, et en mesurer l'impact sur la progression de la perte d'autonomie. De nos jours, plus le patient s'aggrave plus les ressources augmentent. Il est par exemple souvent plus rapide de faire la toilette a un sujet âgé partiellement dépendant que de stimuler ses capacités restantes et faire en sorte qu'il y parvienne de mieux en mieux par lui même. Or même chez les sujets les plus dépendants en institution, il reste des capacités qu'il nous faut développer.

La Gériatrie est aujourd'hui en rupture avec de nombreuses habitudes, dogmes et acquis. Nous avons pris la démarche difficile, de la prévention, de l'évaluation et de la mesure de l'impact de ces programmes. Le but est après les adaptations nécessaires, de les étendre grâce à l'analyse de leur impact, à l'ensemble de la communauté internationale, avec le relais de la société mondiale de gériatrie et bien sur de la SFGG. De telles actions ne sont possibles que grâce au travail quotidien d'hommes et de femmes, et le support de l'ensemble des administrations et collectivités concernées. 